Tôhoku Math. Journ.

21(1969), 548-557.

\title{
NORMAL FAMILIES OF COUSIN I AND II DATA
}

\author{
YUM-TONG SIU*)
}

(Received December 12, 1968; revised February 27, 1969)

It is well-known that on Stein manifolds the solution of the Cousin I problem is always possible and the obstruction to the solution of the Cousin II problem is only topological. Here we use Hörmander's $L^{2}$-estimates for the $\bar{\partial}$ operator to investigate the problem of finding normal families of solutions for given normal families of Cousin I and II data on Stein manifolds. In [8] Stoll proved that, if $M=\boldsymbol{C}^{p}$ with $p>1$, then the following two theorems hold:

(1) Let $N$ be a normal set of non-negative divisors on $M$ and $x_{0} \in M$. Suppose $x_{0}$ dose not belong to the support of any non-negative divisor which is the limit of a net in $N$. Then there exists a continuous map $\gamma$ from $N$ to the set of all holomorphic functions on $M$ such that for $\nu \in N \gamma(\nu)$ defines the divisor $\nu$ and $\gamma(\nu)\left(x_{0}\right)=1$.

(2) Let $N$ be a normal set of non-negative divisors on $M$ and $G$ be an open subset of $M$. Then there is a normal family $\left\{f_{\nu}\right\}_{\nu \in N}$ of holomorphic functions on $M$ such that for $\nu \in N f_{\nu}$ defines the divisor $\nu$ and $f_{\nu}\left(c_{\nu}\right)=1$ for some $c_{\nu} \in G$.

Later (1) and (2) were proved for $M=$ a polydisc by McGrath in [6]. We prove in this paper that on a Stein manifold $M$ a normal family of solutions for a given normal family of Cousin I data can always be found and, if $H^{1}(M, \boldsymbol{R} / \boldsymbol{Z})=H^{2}(M, \boldsymbol{R})=0$, then a normal family of solutions for a given normal family of Cousin II data can always be found. As a consequence, (2) holds for Stein manifolds $M$ satisfying $H^{1}(M, \boldsymbol{R} / \boldsymbol{Z})=H^{2}(M, \boldsymbol{R})=0$ and thus a problem proposed by Stoll (problem 17, p. 307, [1]) is solved for such Stein manifolds. The method we use also enables us to prove (1) for $M=$ the product of $\boldsymbol{C}^{q}$ and the unit ball of $\boldsymbol{C}^{p}$ and a slightly weaker version of (1) for Stein manifolds $M$ satisfying $\pi_{1}(M)=H^{2}(M, \boldsymbol{R})=0$.

In what follows $M$ is a connected positive-dimensional Stein manifold.

*) Supported partially by NSF Grant GP-7265. 
$\mathcal{O}, \mathcal{O}^{*}, \mathscr{M}$, and $\mathscr{M}^{*}$ denote respectively the sheaves of germs of holomorphic, nowhere zero holomorphic, meromorphic, and non-identically-zero meromorphic functions on $M .{ }_{n} \mathcal{O}=$ the structure sheaf of $\boldsymbol{C}^{n}$. If $G$ is an open subset of $M$ (or $\boldsymbol{C}^{u}$ ), then $\boldsymbol{\Gamma}(G, \mathcal{O})$ (or $\boldsymbol{\Gamma}\left(G,{ }_{n} \mathcal{O}\right)$ ), is given the topology of uniform convergence on compact subsets. A normal family in $\mathrm{I}(G, \mathcal{O})$ (or $\mathrm{I}^{\top}\left(G,{ }_{n} \mathcal{O}\right)$ ) is a relatively compact subset of $\Gamma(G, \mathcal{O})\left(\right.$ or $\Gamma\left(G,{ }_{n} \mathcal{O}\right)$ ). $\boldsymbol{N}=$ the set of all natural numbers. If $\boldsymbol{U}=\left\{U_{\lambda}\right\}_{\lambda \in I}$ is an open covering of a topological space, then $U_{\lambda_{0} \cdots \lambda_{p}}$ denotes $U_{\lambda_{0}} \cap \ldots \cap U_{\lambda_{p}},\left\{f_{\lambda_{0} \cdots \lambda_{p}}\right\}_{\lambda_{0}, \cdots, \lambda_{p} \in I}^{\prime}=\left\{f_{\lambda_{0} \cdots \lambda_{p}} \mid \lambda_{0}, \cdots, \lambda_{p} \in I, U_{\lambda_{0} \cdots \lambda_{p}} \neq \emptyset\right\}$, and $\Sigma_{\lambda_{0}, \cdots, \lambda_{p} \in I}^{\prime}=\Sigma_{\lambda_{0}, \cdots, \lambda_{p} \in I, U_{\lambda_{0} \cdots \lambda_{p}} \neq p}$.

Definition 1. Suppose $G$ is an open subset of $M$.

(i) A net $\left\{f_{\sigma}\right\}_{\sigma \in S} \subset \Gamma(G, \mathscr{M})$ is said to converge to $f \in \Gamma(G, \mathscr{M})$ if for every $x \in G$ there exist an open neighborhood $U$ of $x$ in $G$ and $g, h, g_{\sigma}, h_{\sigma} \in$ $\Gamma(U, \mathcal{O}), \sigma \in S$, such that $f_{\sigma}\left|U=g_{\sigma}\left(h_{\sigma}\right)^{-1}, f\right| U=g h^{-1}$, and $g_{\sigma}$ and $h_{\sigma}$ converge respectively to $g$ and $h$ in $\Gamma(U, \mathcal{O})$.

(ii) A normal family $F$ in $\Gamma(G, \mathscr{M})$ is a subset of $\Gamma(G, \mathscr{M})$ such that, if $\left\{f_{\sigma}\right\}_{\sigma \in S}$ is a net in $F$, then there is a subnet $\left\{f_{\sigma(\tau)}\right\}_{\tau \in T}$ converging to some $f \in \mathbf{\Gamma}(G, \mathscr{M})$.

(iii) A normal family $F$ in $\Gamma\left(G, \mathscr{M}^{*}\right)$ is a subset of $\Gamma\left(G, \mathscr{M}^{*}\right)$ such that, if $\left\{f_{\sigma}\right\}_{\sigma \in S}$ is a net in $F$, then there are a subnet $\left\{f_{\sigma(\tau)}\right\}_{\tau \in T}$ and an $f \in \Gamma\left(G, \mathcal{M}^{*}\right)$ with $f_{\sigma(\tau)} \rightarrow f$ in $\Gamma(G, \mathscr{M})$.

\section{REMARKS.}

(i) If $f \in \mathrm{T}(G, \mathcal{O})$ and $\left\{f_{\sigma}\right\}_{\sigma \in S}$ is a net in $\Gamma(G, \mathcal{O})$, then $f_{\sigma} \rightarrow f$ in $\Gamma(G, \mathcal{O})$ if and only if $f_{\sigma} \rightarrow f$ in $\Gamma(G, \mathscr{M}$ ) (Lemma 1 below).

(ii) The convergence defined for $\mathrm{T}(G, \mathscr{M})$ in Def. 1 (i) does not define a topology for $\Gamma(G, \mathscr{M})$ corresponding to it as is seen in the following counterexample: On $\boldsymbol{C}$ define meromorphic functions $f(z) \equiv 1$,

$$
f_{n}(z) \equiv 1, \quad f_{n, m}(z)=\left(z+\frac{1}{m}\right)^{n}\left(z-\frac{1}{m}\right)^{-n}
$$

$m, n \in \boldsymbol{N}$. Let $S=\{(n, h) \mid n \in \boldsymbol{N}, h$ is a map from $\boldsymbol{N}$ to $\boldsymbol{N}\}$ be directed by the following ordering: $(n, h) \leqq\left(n^{\prime}, h^{\prime}\right)$ if and only if $n \leqq n^{\prime}$ and $h(m) \leqq h^{\prime}(m)$ for all $m \in \boldsymbol{N}$. Let $g_{(n, h)}=f_{n, h(n)}$ for $(n, h) \in S$. Then $f_{n} \rightarrow f$ as $n \rightarrow \infty$ and for fixed $n \in \boldsymbol{N} f_{n, m} \rightarrow f_{n}$ as $m \rightarrow \infty$. However, the net $\left\{g_{(n, h)}\right\}_{(n, h) \in S}$ does not converge to $f$. 


\section{DEFINITION 2.}

(i) A Cousin I datum $f$ on $M$ is an element of $I^{\prime}(M, \mathcal{M} / \mathcal{O}$ ), where $\mathcal{O}$ is regarded as a sulssheaf of the additive sheaf $\mathscr{M}$.

(ii) $\Lambda$ solution of a Cousin I datum $f$ on $M$ is an element of $I^{\prime}(M, \mathcal{M})$ which is mapped to $f$ under the quotient map $\mathscr{M} \rightarrow \mathscr{M} / \mathcal{O}$.

(iii) A normal family of Cousin I data $\left\{f^{(\alpha)}\right\}_{\alpha \in A}$ is a set of Cousin I data on $M$ with the following property: for every point $x$ of $M$ there exist an open neighborhood $U$ of $x$ in $M$ and a normal family $\left\{g^{(\alpha)}\right\}_{\alpha \in A}$ in $\Gamma(U, \mathscr{M})$ such that $g^{(\alpha)}$ is a solution of $f^{(\alpha)} \mid U$ for $\alpha \in A$.

\section{DEFINITION 3.}

(i) A Cousin II datum (or a divisor) $f$ on $M$ is an element of $\Gamma\left(M, \mathscr{M}^{*} / \mathcal{O}^{*}\right)$, where $\mathcal{O}^{*}$ is regarded as a subsheaf of the multiplicative sheaf $\mathscr{M}^{*}$. $f$ is called non-negative if every point of $M$ has a connected open neighborhood $U$ such that $f \mid U$ is the image of some non-zero element cf $\Gamma(U, \mathcal{O})$ under the quotient map $\mathscr{M}^{*} \rightarrow \mathscr{M}^{*} / \mathcal{O}^{*}$.

(ii) A solution of a Cousin II datum $f$ on $M$ is an element of $\Gamma\left(M, \mathscr{M}^{*}\right)$ which is mapped to $f$ under the quotient map $\mathscr{M}^{*} \rightarrow \mathscr{M}^{*} / \mathcal{O}^{*}$.

(iii) A normal family of Cousin II data $\left\{f^{(\alpha)}\right\}_{\alpha \in A}$ is a set of Cousin II data on $M$ with the following property: for every point $x$ of $M$ there exist an open neighborhood $U$ of $x$ in $M$ and a normal family $\left\{g^{(\alpha)}\right\}_{\alpha \in A}$ in $\Gamma^{\prime}\left(U, \mathscr{M}^{*}\right)$ such that $g^{(\alpha)}$ is a solution of $f^{(\alpha)} \mid U$ for $\alpha \in A$.

REMARK. The definition given here of a normal family of non-negative Cousin II data agrees with the definition given in [8] of a normal family of non-negative divisors.

DEFINITION 4. A normal family of non-negative divisors $F$ on $M$ is called small if we can find an open covering $\boldsymbol{U}=\left\{U_{\lambda}\right\}_{\lambda \in I}$ of $M$ and a subset $K$ of $M$ such that (1) intersections of finite subcollections of $\boldsymbol{U}$ are empty or contractible open subsets of $M,(2) U_{i}^{-}$is a compact subset of an open subset of $M$ which is biholomorphic to a ball in a complex number space, (3) $K \cap U_{\lambda \mu} \neq \emptyset$ if $U_{\lambda} \cap U_{\mu} \neq \emptyset$, (4) $K \cap U_{\lambda}$ is pathwise connected for $\lambda \in I$, and (5) if a non-negative divisor $f$ on $M$ is the limit of a net in $F$, then $K \cap \operatorname{Supp} f=\emptyset$ (where the set of non-negative divisors is given the topology defined in [8] and Supp $f$ denotes the support of the section $f$ of the sheaf $\mathscr{M}^{*} / \mathcal{O}^{*}$ on $M$ ). 
The following three theorems are the main results:

THEOREM 1. Every normal family of Cousin I data on M admits a family of solutions which is normal in $\mathrm{r}(M, \mathcal{M})$.

THEOREM 2. If $H^{1}(M, \boldsymbol{R} / \boldsymbol{Z})=H^{2}(M, \boldsymbol{R})=0$, then every normal family of Cousin II data on $M$ admits a family of solutions which is normal in $\Gamma\left(M, \mathscr{M}^{*}\right)$.

THEOREM 3. If $\pi_{1}(M)=H^{2}(M, \boldsymbol{R})=0$ and $F$ is a small normal family of non-negative divisors on $M$, then there is a continuous map $\gamma: F \rightarrow \Gamma(M, \mathcal{O})$ such that $\gamma(f)$ is a solution of $f$ for every $f \in F$.

REMARKS. (i) Suppose $G$ is an open subset of $M$. Th. 2 implies that every normal family of non-negative divisors admits a normal family of solutions $F$ such that for every $f \in F$ there exists $x \in G$ with $f(x)=1$. The reason is the following: For the given family of non-negative divisors we first find a normal family of solutions $H$. For $x \in G$ and $n \in \boldsymbol{N}$ let $D_{x, n}=\{f \in \Gamma(M, \mathcal{O})|| f(x) \mid>1 / n\}$. Since the closure $H^{-}$of $H$ in $\Gamma(M, \mathcal{O})$ is compact and $H^{-} \subset \cup\left\{D_{x, n} \mid x \in G, n \in \boldsymbol{N}\right\}$, $H^{-} \subset \cup_{i=1}^{k} D_{x_{i}, n_{i}}$ for some $x_{1}, \cdots, x_{k} \in G$ and $n_{1}, \cdots, n_{k} \in \boldsymbol{N}$. For $h \in H \quad h \in D_{x_{i}, n_{i}}$ for some $i$. Define $f_{h}=h\left(x_{i}\right)^{-1} h$. The normal family of solutions $F=\left\{f_{h}\right\}_{h \in H}$ satisfies the requirement.

(ii) Under the assumptions of Th. 3, if $x_{0} \in M$ such that $x_{0} \notin$ Supp $f$ for $f \in F^{-}$, then we can choose $\gamma$ such that $\gamma(f)\left(x_{0}\right)=1$. The reason is the following: We first find a continuous map $\tilde{\gamma}: F^{-} \rightarrow \Gamma(M, \mathcal{O})$ such that $\tilde{\gamma}(f)$ is a solution of $f$. Then define $\gamma: F \rightarrow \Gamma(M, \mathcal{O})$ by $\gamma(f)=f\left(x_{0}\right)^{-1} \tilde{\gamma}(f)$.

LemMA 1. Suppose $G$ is a connected open subset of $M$. Suppose $f, g \neq 0 \in \Gamma(G, \mathcal{O})$ and $\left\{f_{\sigma}\right\}_{\sigma \in S}, \quad\left\{g_{\sigma}\right\}_{\sigma \in S}$ are nets in $\Gamma(G, \mathcal{O})$ such that $(1)$ $f_{\sigma}\left(g_{\sigma}\right)^{-1} \in \mathrm{I}(G, \mathcal{O}), \sigma \in S$, and $(2) f_{\sigma} \rightarrow f$ and $g_{\sigma} \rightarrow g$ in $\mathrm{\Gamma}(G, \mathcal{O})$. Then $f g^{-1} \in \Gamma(G, \mathcal{O})$ and $f_{\sigma}\left(g_{\sigma}\right)^{-1} \rightarrow f g^{-1}$ in $\Gamma(G, \mathcal{O})$.

PROOF. Let $Z$ be the zero-set of $g$. We have to prove that every point of $G$ has an open neighborhood in which $\mathrm{fg}^{-1}$ is holomorphic and on which $f_{\sigma}\left(g_{\sigma}\right)^{-1}$ converges uniformly to $f g^{-1}$. Fix $z^{0} \in G$. If $z^{0} \notin Z$, then $f_{\sigma}\left(g_{\sigma}\right)^{-1}$ converges uniformly to $f g^{-1}$ on any compact neighborhood of $z^{0}$ in $G$ disjoint from $Z$. Suppose $z^{0} \in Z$. We can assume w. l.o.g. that (1) $G$ is an open subset of $\boldsymbol{C}^{n}$, (2) $z^{0}=0$, (3) $K=\left\{\left(z_{1}, \cdots, z_{n}\right) \in \boldsymbol{C}^{n}|| z_{i} \mid \leqq 1,1 \leqq i \leqq n\right\} \subset G$, and (4) $L=\left\{\left(z_{1}, \cdots, z_{n}\right) \in K|| z_{n} \mid=1\right\}$ is disjoint from $Z$. Then $f_{\sigma}\left(g_{\sigma}\right)^{-1}$ converges uniformly to $f g^{-1}$ on $L$. By considering the coefficients of the negative powers of $z_{n}$ in the Laurent series of $\mathrm{fg}^{-1}$ with respect to $z_{n}$, we conclude that 
$f g^{-1}$ is holomorphic in the interior of $K$. Hence $f_{\sigma}\left(g_{\sigma}\right)^{-1}$ converges to $f g^{-1}$ uniformly on the interior of $K$. q.e.d.

Lemma 2. Suppose $G$ is an open subset of $M$ and $K$ is a compact subset of $G$. If $F$ is a normal family in $\mathrm{r}(G, \mathcal{O})$, then $F$ is uniformly bounded on $K$.

Proof. This follows from the fact that the map $\Phi: \Gamma(G, \mathcal{O}) \rightarrow[0, \infty)$ defined by $\Phi(f)=|f(K)|$ for $f \in \Gamma(G, \mathcal{O})$ is upper semi-continuous. q. e. d.

LEMMA 3. Suppose $\left\{a_{n}\right\}_{n \in N}$ and $\left\{b_{n}\right\}_{n \in N}$ are two non-decreasing sequences of points in $[0, \infty)$ such that $a_{n} \rightarrow \infty$. Then there exists a $C^{2}$ real-valued function $\rho$ on $[0, \infty)$ such that $\rho^{\prime} \geqq 0, \rho^{\prime \prime} \geqq 0$, and $\rho\left(a_{n}\right) \geqq b_{n}$ for $n \in \boldsymbol{N}$ (where $\rho^{\prime}$ and $\rho^{\prime \prime}$ are respectively the first and second derivatives of $\rho$ ).

PROOF. Define $\rho_{0}$ on $[0, \infty)$ by $\rho_{0}(x)=b_{n}$ for $a_{n-1} \leqq x<a_{n}$ (where $\left.a_{-1}=0\right)$. Define $\rho_{i+1}(x)=\int_{0}^{x+1} \rho_{i}(t) d t$ for $0 \leqq i \leqq 2$. Then $\rho=\rho_{3}$ satisfies the requirement. q. e. d.

LEMMA 4. Suppose $\Omega$ is a connected Stein open subset of $\boldsymbol{C}^{N}$ and $\left\{K_{n}\right\}_{n \in N}$ is a locally finite sequence of compact subsets of $\Omega$. Suppose $\left\{c_{n}\right\}_{n \in N} \subset[0, \infty)$. Then there exists a $C^{2}$ plurisubharmonic function $\phi$ on $\Omega$ such that inf $\left\{\phi(z) \mid z \in K_{n}\right\} \geqq c_{n}, n \in \boldsymbol{N}$.

ProOF. Let $f: \Omega \rightarrow \boldsymbol{C}^{N^{\prime}}$ be an embedding of $\Omega$ as a closed complex submanifold of $\boldsymbol{C}^{N^{\prime}}$. For $n \in \boldsymbol{N}$ let $a_{n}$ be the largest integer such that $f\left(\bigcup_{m=n}^{\infty} K_{m}\right)$ is disjoint from the ball of $\boldsymbol{C}^{N^{\prime}}$ centered at 0 and with radius $a_{n}$. Since $\left\{K_{n}\right\}_{n \in N}$ is locally finite, $a_{n} \rightarrow \infty$. Let $b_{n}=\sup _{1 \leqq m \leqq n} c_{m}, n \in \boldsymbol{N}$. By Lemma 3 there exists a $C^{2}$ real-valued function $\rho$ on $[0, \infty)$ such that $\rho^{\prime} \geqq 0, \rho^{\prime \prime} \geqq 0$, and $\rho\left(a_{n}^{2}\right) \geqq b_{n}, n \in \boldsymbol{N}$. Define $\widetilde{\boldsymbol{\varphi}}(z)=\rho\left(|z|^{2}\right)$ for $z \in \boldsymbol{C}^{N^{\prime}}$. Since $\sum_{i, j=1}^{N^{\prime}} \frac{\partial^{2} \widetilde{\varphi}}{\partial z_{i}} \bar{\partial} \bar{z}_{j}(z) \xi_{i} \bar{\xi}_{j}=\rho^{\prime \prime}\left(|z|^{2}\right)\left|\sum_{i=1}^{N^{\prime}} \xi_{i} \bar{z}_{i}\right|^{2}$ $+\rho^{\prime}\left(|z|^{2}\right)\left(\sum_{i=1}^{N^{\prime}}\left|\xi_{i}\right|^{2}\right) \geqq 0$ for $\xi_{1}, \cdots, \xi_{N^{\prime}} \in \boldsymbol{C}, \quad \widetilde{\Phi}$ is plurisubharmonic on $\boldsymbol{C}^{N^{\prime}}$. $\phi=\widetilde{\boldsymbol{\phi}} \circ f$ satisfies the requirement. q. e.d.

Lemma 5. Suppose $H_{1}$ and $H_{2}$ are Hilbert spaces and $\phi: H_{1} \rightarrow H_{2}$ is a continuous linear surjection. Then there exists a continuous linear map $\psi$ : $H_{2} \rightarrow H_{1}$ such that $\phi \circ \psi=$ the identity map on $H_{2}$. 
Proof. Let $L$ be the orthogonal complement of $\operatorname{Ker} \phi$ in $H_{1}$. Define $\psi: H_{2} \rightarrow H_{1}$ as follows: for $x \in H_{2}$ set $\psi(x)$ to be the unique element in $L$ such that $\psi(\psi(x))=x$. The open mapping theorem implies that $\psi$ is continuous. q.e.d.

Lemma 6. Suppose $E$ and $F$ are Fréchet spaces and $\phi: E \rightarrow F$ is a continuous linear surjection. If $K$ is a compact subset of $F$, then there exists a compact subset $L$ of $E$ such that $\varphi(L)=K$.

PROOF. This follows from $\$ 22.2(7)$, p. 281, [5] and the open mapping theorem. q. e. d.

Lemma 7. Suppose $H^{2}(M, \boldsymbol{R})=0$ and $\boldsymbol{U}=\left\{U_{\lambda}\right\}_{\lambda \in N}$ is an open covering of $M$ such that intersections of finite subcollections of $\boldsymbol{U}$ are empty or contractible open subsets of $M$. Suppose $K$ is a compact subset of $Z^{2}(\boldsymbol{U}, \boldsymbol{R})$.

(i) There exists a continuous linear map $\phi: K \rightarrow C^{1}(\boldsymbol{U}, \boldsymbol{R})$ such that $\delta \circ \phi=$ the identity map on $K$.

(ii) If in addition $K \subset Z^{2}(\boldsymbol{U}, \boldsymbol{Z})$ and $H^{\prime}(M, \boldsymbol{R} / \boldsymbol{Z})=0$, then there exists a compact subset. $L$ of $C^{1}(\boldsymbol{U}, \boldsymbol{Z})$ such that $\delta(L)=K$.

ProOF. (i) There exists $\xi_{\lambda \mu \nu}>0$ for $U_{\lambda \mu \nu} \neq \emptyset$ such that

$$
\left|a_{\lambda \mu \nu}\right| \leqq \xi_{\lambda \mu \nu} \text { for }\left\{a_{\lambda \mu \nu}\right\}_{\lambda, \mu, \nu \in N}^{\prime} \in K
$$

Since $\delta: C^{1}(\boldsymbol{U}, \boldsymbol{R}) \rightarrow Z^{2}(\boldsymbol{U}, \boldsymbol{R})$ is surjective, by Lemma 6 there exists $\eta_{\lambda \mu}>0$ for $U_{\lambda \mu} \neq \emptyset$ such that, if $a=\left\{a_{\lambda \mu \nu}\right\}_{\lambda, \mu, \nu \in N}^{\prime} \in Z^{2}(\boldsymbol{U}, \boldsymbol{R})$ and $\left|a_{\lambda \mu \nu}\right| \leqq 2^{\frac{\lambda+\mu+\nu}{2}} \xi_{\lambda \mu \nu}$, then there exists $b=\left\{b_{\lambda \mu}\right\}_{\lambda, \mu \in N}^{\prime} \in C^{1}(\boldsymbol{U}, \boldsymbol{R})$ with $\delta b=a$ and $\left|b_{\lambda \mu}\right| \leqq \eta_{\lambda \mu}$. Let $H_{1}=\{b$ $\left.=\left.\left\{b_{\lambda \mu}\right\}_{\lambda, \mu \in N}^{\prime} \in C^{1}(\boldsymbol{U}, \boldsymbol{R})\left|\Sigma_{\lambda, \mu \in N}^{\prime} 2^{-\lambda-\mu} \eta_{\lambda \mu}^{-2}\right| b_{\lambda \mu}\right|^{2}+\Sigma_{\lambda, \mu \nu \in N}^{\prime} 2^{-\lambda-\mu-\nu} \xi_{\lambda \mu \nu}^{-2}\left|(\delta b)_{\lambda \mu \nu}\right|^{2}<\infty\right\}$ and $H_{2}=\left\{a=\left.\left\{a_{\lambda \mu \nu}\right\}_{\lambda, \mu, \nu \in N}^{\prime} \in Z^{2}(\boldsymbol{U}, \boldsymbol{R})\left|\|a\|^{2}=\sum_{\lambda, \mu, \nu \in N}^{\prime} 2^{-\lambda-\mu-\nu} \xi_{\lambda \mu \nu}^{-2}\right| a_{\lambda \mu \nu}\right|^{2}<\infty\right\} . H_{1}$ and $H_{2}$ are Hilbert spaces. We claim that the map $\varphi_{1}: H_{1} \rightarrow H_{2}$ induced by $\delta$ is surjective. Fix $a=\left\{a_{\lambda \mu \nu}\right\}_{\lambda, \mu, \nu \in N}^{\prime} \in H_{2}$ and we want to find $b \in H_{1}$ such that $\phi_{1}(b)=a$. We can assume w. 1. o. g that $\|a\| \leqq 1$. Then $\left|a_{\lambda \mu \nu}\right| \leqq 2^{\frac{\lambda+\mu+\nu}{2}} \xi_{\lambda \mu \nu}$. There exists $b=\left\{b_{\lambda \mu}\right\}_{\lambda, \mu \in N}^{\prime} \in C^{1}(\boldsymbol{U}, \boldsymbol{R})$ with $\delta b=a$ and $\left|b_{\lambda \mu}\right| \leqq \eta_{\lambda \mu}$. Hence $b \in H_{1}$ and $\phi_{1}(b)=a$. By Lemma 5 there exists a continuous linear map $\varphi_{2}: H_{2} \rightarrow H_{1}$ such that $\varphi_{1} \varphi_{2}=$ the identity map on $H_{2}$. Let $i_{1}: K \rightarrow H_{2}$ and $i_{2}: H_{1} \rightarrow C^{1}(\boldsymbol{U}, \boldsymbol{R})$ be inclusion maps. $i_{1}$ is continuous because of (3). $\phi=i_{2} \phi_{2} i_{1}$ satisfies the requirement.

(ii) Let $G$ be the multiplicative group $\{z \in \boldsymbol{C}|| z \mid=1\}$. Since $G \approx \boldsymbol{R} / \boldsymbol{Z}$, $H^{\prime}(M, G)=0 . \quad$ Let $K=\left\{a^{(\alpha)}\right\}_{\alpha \in A} . \quad$ Let $\varphi\left(a^{(\alpha)}\right)=b^{(\alpha)}=\left\{b_{\lambda \mu}^{(\alpha)}\right\}_{\lambda, \mu \in N .}^{\prime} \quad \exp \left(2 \pi i b_{\lambda \mu \nu}^{(\alpha)}\right)$ $\exp \left(2 \pi i b_{\mu \nu}^{(\alpha)}\right) \exp \left(2 \pi i b_{\lambda \nu}^{(\alpha)}\right)=1$ for $U_{\lambda \mu \nu} \neq \emptyset$. Hence there exists $0 \leqq b_{\lambda}^{(\alpha)}<1$ for $U_{\lambda}$ 
$\neq \emptyset$ and $\alpha \in A$, such that for $U_{\lambda \mu} \neq \emptyset \exp \left(2 \pi i b_{\mu}^{(\alpha)}\right) \exp \left(-2 \pi i b_{\lambda}^{(\alpha)}\right)=\exp \left(2 \pi i b_{\lambda \mu}^{(\alpha)}\right)$. Let $c_{\lambda \mu}^{(\alpha)}=b_{\lambda \mu}^{(\alpha)}+b_{\lambda}^{(\alpha)}-b_{\mu}^{(\alpha)}$. Then $c^{(\alpha)}=\left\{c_{\lambda \mu}^{(\alpha)}\right\}_{\lambda, \mu \in N}^{\prime} \in C^{1}(\boldsymbol{U}, \boldsymbol{Z}), \alpha \in A$. The closure $L$ of $\left\{c^{(\alpha)}\right\}_{\alpha \in A}$ in $C^{1}(\boldsymbol{U}, \boldsymbol{Z})$ satisfies the requirement. q. e. d.

Lemma 8. Suppose $\Omega$ is a Stein open subset of $\boldsymbol{C}^{N}$. Suppose $\boldsymbol{U}=\left\{U_{\lambda}\right\}_{\lambda \in I}$ is a locally finite open covering of $\Omega$ such that $U_{\lambda}$ is relatively compact in $\Omega, \lambda \in I$. Suppose $F \subset Z^{1}(\boldsymbol{U}, \mathcal{O})$ and $a_{\lambda \mu}>0, \lambda, \mu \in I$, such that $\left|f_{\lambda \mu}\left(U_{\lambda \mu}\right)\right| \leqq a_{\lambda \mu}$ for $\left\{f_{\lambda \mu}\right\}_{\lambda, \mu \in I}^{\prime} \in F$. Then there exists a continuous map $\gamma: F \rightarrow C^{0}(\boldsymbol{U}, \mathcal{O})$ such that $\delta(\gamma(f))=f$ for $f \in F$.

PROOF. Let $\left\{\rho_{\lambda}\right\}_{\lambda \in I}$ be a partition of unity subordinate to $\boldsymbol{U}$. Let $b_{\lambda}=\sup _{1 \leqq i \leqq N}\left|\frac{\partial \rho_{\lambda}}{\partial \bar{z}_{i}}(\Omega)\right|$ and $c_{\lambda}=\Sigma\left\{b_{\mu} a_{\mu \lambda} \mid U_{\lambda} \cap U_{\mu} \neq \emptyset\right\}, \lambda \in I$. Let $k$ be a $C^{\infty}$ positivevalued function on $\Omega$ such that $e=\int_{\Omega} k d x<\infty$ (where $d x$ is the Euclidean volume element of $\left.\boldsymbol{C}^{N}\right)$. Since $\Omega$ is $\boldsymbol{\sigma}$-compact, $\left\{\lambda \in I \mid U_{\lambda} \neq \emptyset\right\}$ is countable. By Lemma 4 there is a $C^{2}$ plurisubharmonic function $\phi$ on $\Omega$ such that inf $\left\{\varphi(z) \mid z \in U_{\lambda}^{-}\right\} \geqq \log \left(N c_{\lambda}^{2}\right)+\left|\left(\log k^{-1}\right)\left(U_{\lambda}^{-}\right)\right|$for $U_{\lambda} \neq \emptyset$. Let $\psi(z)=\psi(z)$ $+2 \log \left(1+|z|^{2}\right)$ on $\Omega$. Let $H_{1}=\{\eta \mid \eta$ is a locally square integrable function on $\Omega$ and $\overline{\bar{\partial}} \eta$ is an $(0,1)$-form on $\Omega$ with locally square integrable functions as coefficients such that $\left.\|\eta\|_{\psi}^{2}+\|\bar{\partial} \eta\|_{\varphi}^{2}<\infty\right\}$ and let $H_{2}=\{\omega \mid \omega$ is a $(0,1)$-form on $\Omega$ with locally square integrable functions as coefficients such that $\bar{\partial} \omega=0$ and $\left.\|\omega\|_{\rho}<\infty\right\}$, where $\bar{\partial}$ is in the distribution sense and the norms $\|\cdot\|_{\boldsymbol{\rho}}$ and $\|\cdot\|_{\psi}$ are as defined on pp. 77-78 of [3]. $H_{1}$ and $H_{2}$ are Hilbert spaces. We are going to define a map $\gamma_{1}: F \rightarrow H_{2}$. Take $f=\left\{f_{\mu \nu}\right\}_{\mu, \nu \in I}^{\prime} \in F$. Let $h_{\lambda}^{(f)}=\Sigma_{\mu} \sigma_{\mu}$, where $\sigma_{\mu}$ is the trivial extension of $\rho_{\mu} f_{\mu \lambda}$ on $U_{\mu}$. Then $f_{\lambda \mu}=h_{\mu}^{(f)}-h_{\lambda}^{(f)}$ on $U_{\lambda \mu}$. $\quad 0=\bar{\partial} h_{\mu}^{(f)}$ $-\bar{\partial} h_{\lambda}^{(f)}$ on $U_{\lambda \mu}$. There is a unique $C^{\infty}(0,1)$-form $\omega$ on $\Omega$ such that $\omega=\bar{\partial} h_{\lambda}^{(f)}$ on $U_{\lambda}$. Then $\bar{\partial} \omega=0$ and $\|\omega\|_{\varphi} \leqq \sqrt{e}$. Hence $\omega \in H_{2}$. Set $\gamma_{1}(f)=\omega$. $\quad \gamma_{1}$ is continuous.

Let $\theta: H_{1} \rightarrow H_{2}$ be induced by $\bar{\partial}$.

Then $\theta$ is a continuous linear surjection (Lemma 4, p. 945, [4]). By Lemma 5 there exists a continuous linear map $\gamma_{2}: H_{2} \rightarrow H_{1}$ such that $\theta \circ \gamma_{2}=$ the identity map on $H_{2}$. Define $\gamma: F \rightarrow C^{0}(\boldsymbol{U}, \mathcal{O})$ as follows: Take $f \in F$. Let $g_{\lambda}=h_{\lambda}^{(f)}-\gamma_{2}\left(\gamma_{1}(f)\right) \mid U_{\lambda}$, where $h_{\lambda}^{(f)}$ is as defined above. Set $\gamma(f)=\left\{g_{\lambda}\right\}_{\lambda \in I}^{\prime}$. Then $\gamma$ satisfies the requirement. q. e. d.

THEOREM 4. Suppose $\boldsymbol{U}=\left\{U_{\lambda}\right\}_{\lambda \in I}$ and $\boldsymbol{V}=\left\{V_{\lambda}\right\}_{\lambda \in I}$ are open coverings of $M$ such that $\boldsymbol{V}$ is locally finite and $V_{\lambda}^{-}$is a compact subset of $U_{\lambda}, \lambda \in I$. Suppose $F$ is a compact subset of $Z^{1}(\boldsymbol{U}, \mathcal{O})$. Then there exists a continuous map $\gamma: F \rightarrow C^{\prime \prime}(\boldsymbol{V}, \mathcal{O})$ such that $\delta(\gamma(f))=f$ on $\boldsymbol{V}$.

Proof. $M$ is a closed complex submanifold of $\boldsymbol{C}^{N}$ for some $N$. There 
exists an open neighborhood $\Omega_{1}$ of $M$ in $C^{N}$ and a holomorphic retraction $\rho_{1}$ : $\Omega_{1} \rightarrow M$ (VIII. C. 8., p. 257, [2]). There exists a Stein open neighborhood $\Omega$ of $M$ in $\Omega_{1}$ (Th. 2, p. 380, [7]). Let $\rho=\rho_{1} \mid \Omega$. Let $\widetilde{\boldsymbol{V}}=\left\{\widetilde{V}_{\lambda}\right\}_{\lambda \in I}$, where $\widetilde{V}_{\lambda}=\rho^{-1}\left(V_{\lambda}\right)$. Let $\rho^{*}: Z^{1}\left(\boldsymbol{U},(\mathcal{\Theta}) \rightarrow Z^{1}\left(\widetilde{\boldsymbol{V}},{ }_{N} \mathcal{\Theta}\right)\right.$ be induced by $\rho$, i. e., if $\left\{f_{\lambda \mu}\right\}_{\lambda, \mu \in I}^{\prime} \in Z^{1}(\boldsymbol{U}, \mathcal{O})$, then $\rho^{*}(f)=\left\{\widetilde{f}_{\lambda \mu}\right\}_{\lambda, \mu \in I}^{\prime} \in Z^{1}\left(\widetilde{\boldsymbol{V}},{ }_{N} \mathcal{O}\right)$, where $\widetilde{f}_{\lambda \mu}=\left(f_{\lambda \mu} \circ \rho\right) \mid \widetilde{V}_{\lambda \mu} . \rho^{*}$ is linear and continuous. Let $F=\rho^{*}(\widetilde{F})$. By Lemma 2 there exists $a_{\lambda \mu}>0, \lambda, \mu \in I$, such that $\left|f_{\lambda \mu}\left(V_{\lambda \mu}^{-}\right)\right| \leqq a_{\lambda \mu}$ for $\left\{f_{\lambda \mu}\right\}_{\lambda, \mu \in I}^{\prime} \in F$. Hence $\left|\widetilde{f}_{\lambda \mu}\left(\widetilde{V}_{\lambda \mu}\right)\right| \leqq a_{\lambda \mu}$ for $\left\{\widetilde{f}_{\lambda \mu}\right\}_{\lambda, \mu \in I}^{\prime} \in \widetilde{F}$. By Lemma 8 there exists a continuous map $\tilde{\gamma}: \quad \widetilde{F} \rightarrow C^{0}\left(\widetilde{\boldsymbol{V}},{ }_{N} \Theta\right)$ such that $\delta(\widetilde{\gamma}(\widetilde{f}))=\widetilde{f}$ for $\widetilde{f} \in \widetilde{F}$. Define $\gamma: F \rightarrow C^{0}(\boldsymbol{V}, \mathcal{O})$ as follows: Take $f \in F$. Let $\tilde{\gamma}\left(\rho^{*}(f)\right)=\left\{\tilde{g}_{k}\right\}_{\lambda \in I}^{\prime}$. Let $g_{\lambda}=\widetilde{g}_{\lambda} \mid V_{\lambda}$. Set $\gamma(f)=\left\{g_{\lambda}\right\}_{\lambda \in I}^{\prime}$. Then $\gamma$ satisfies the requirement. q. e.d.

REMARK. The map $\gamma$ constructed in the proofs of Lemma 8 and Th. 4 satisfies the following linearity conditions: $\gamma\left(f+f^{\prime}\right)=\gamma(f)+\gamma\left(f^{\prime}\right)$ and $\gamma(a f)=a \gamma(f)$ if $a \in \boldsymbol{C}$ and $f, f^{\prime}, f+f^{\prime}$, af $\in F$.

Proof of Theorem 1. Suppose $F=\left\{f^{(\alpha)}\right\}_{\alpha \in A}$ is a normal family of Cousin I data on $M$. There exists an open covering $\boldsymbol{U}=\left\{U_{\lambda}\right\}_{\lambda \in I}$ of $M$ and $\left\{f_{\lambda}^{(\alpha)}\right\}_{\lambda \in I}^{\prime} \in C^{0}(\boldsymbol{U}, \mathscr{M}), \alpha \in A$, such that (1) $f_{\lambda}^{(\alpha)}$ is a solution of $f^{(\alpha)} \mid U_{\lambda}, \alpha \in A$, $\lambda \in I$, and (2) for fixed $\lambda \in I\left\{f_{\lambda}^{(\alpha}\right\}_{\alpha \in A}$ is a normal family in $\Gamma\left(U_{\lambda}, \mathscr{M}\right)$. We can suppose w. l.o.g. that $\boldsymbol{U}$ is locally finite and there is an open covering $\boldsymbol{V}=\left\{V_{\lambda}\right\}_{\lambda \in I}$ of $M$ such that $V_{\lambda}^{-}$is a compact subset of $U_{\lambda}, \lambda \in I$.

Let $h_{\lambda \mu}^{(\alpha)}=\left(f_{\mu}^{(\alpha)}-f_{\lambda}^{(\alpha)}\right) \mid U_{\lambda \mu}, \alpha \in A, \lambda, \mu \in I . \quad h^{(\alpha)}=\left\{h_{\lambda \mu}^{(\alpha)}\right\}_{\lambda, \mu \in I}^{\prime} \in Z^{\mathrm{I}}(\boldsymbol{U}, \mathcal{O}) . \quad$ By Lemma 1 for fixed $\lambda, \mu \in I\left\{h_{\lambda \mu}^{(\alpha)}\right\}_{\alpha \in A}$ is a normal family in $\Gamma\left(U_{\lambda \mu}, \mathcal{O}\right)$. By Th. 4 there exists $k^{(\alpha)}=\left\{k_{\lambda}^{(\alpha)}\right\}_{\lambda \in I}^{\prime} \in C^{0}(\boldsymbol{V}, \mathcal{O}), \alpha \in A$, such that $\delta k^{(\alpha)}=h^{(\alpha)}$ on $\boldsymbol{V}, \alpha \in A$, and for fixed $\lambda \in I\left\{k_{\lambda}^{(\alpha}\right\}_{\alpha \in A}$ is a normal family in $\Gamma\left(V_{\lambda}, \mathcal{O}\right)$. Define $g^{(\alpha)} \in \Gamma(M, \mathcal{M})$, $\alpha \in A$, by setting $g^{(\alpha)}=f_{\lambda}^{(\alpha)}-k_{\lambda}{ }^{\alpha)}$ on $V_{\lambda}, \alpha \in A . \quad\left\{g^{(\alpha)}\right\}_{\alpha \in A}$ is a normal family in $\Gamma(M, \mathscr{M})$ and $g^{(\alpha}$ is a solution of $f^{(\alpha)}, \alpha \in A$. q. e. d.

Proof of Theorem 2. Suppose $F=\left\{f^{(\alpha)}\right\}_{\alpha \in A}$ is a normal family of Cousin II data on $M$. There exist an open covering $\boldsymbol{U}=\left\{U_{\lambda}\right\}_{\lambda \in I}$ of $M$ and $\left\{f_{\lambda}^{(\alpha)}\right\}_{\lambda \in I}^{\prime} \in C^{0}\left(\boldsymbol{U}, \mathcal{M}^{*}\right), \alpha \in A$, such that $(1) f_{\lambda}^{(\alpha)}$ is a solution of $f^{(\alpha)} \mid U_{\lambda}, \alpha \in A$, $\lambda \in I$, and (2) for fixed $\lambda \in I\left\{f_{\lambda}^{(\alpha)}\right\}_{\alpha \in A}$ is a normal family in $\Gamma\left(U_{\lambda}, \mathscr{M}^{* *}\right)$. We can suppose w. l.o.g. that (1) intersections of finite subcollections of $\boldsymbol{U}$ are empty or contractible open subsets of $M$, (2) $\boldsymbol{U}$ is locally finite, and $I=\boldsymbol{N}$ and (3) there exists an open covering $\boldsymbol{V}=\left\{V_{\lambda}\right\}_{\lambda \in I}$ of $M$ such that $V_{\lambda}^{-}$is a compact subset of $U_{\lambda}, \lambda \in I$.

Let $h_{\lambda \mu}^{(\alpha)}=f_{\mu}^{(\alpha)}\left(f_{\lambda}^{\alpha)}\right)^{-1} \mid U_{\lambda l}, \alpha \in A, \lambda, \mu \in I . \quad$ By Lemma 1 for fixed $\lambda, \mu$ $\in I\left\{h_{\lambda \mu}^{(\alpha)}\right\}_{\alpha \in A}$ is a relatively compact subset of $\Gamma^{\prime}\left(U_{\lambda \mu}, \mathcal{C}^{*}\right)$ (where $\Gamma^{\top}\left(U_{\lambda \mu}, \mathcal{O}^{*}\right)$ is given the topology induced from $\mathrm{\Gamma}\left(U_{\lambda \mu}, \mathcal{O}\right)$ ). Fix $x_{\lambda \mu} \in U_{\lambda \mu}$ for $U_{\lambda \mu} \neq \emptyset$. Since $U_{\lambda \mu}$ is empty or contractible, for $U_{\lambda \mu} \neq \emptyset$ and $\lambda<\mu$ there exists a unique 
$k_{\lambda \mu}^{(\alpha)} \in \Gamma\left(U_{\lambda \mu}, C\right)$ such that $\exp \left(2 \pi i k_{\lambda \mu}^{(\alpha)}\right)=h_{\lambda \mu}^{(\alpha)}$ and the real part of $k_{\lambda \mu}^{(\alpha)}\left(x_{\lambda \mu}\right)$ is in $\left[\begin{array}{ll}0 & 1\end{array}\right)$. Set $k_{\mu \lambda}^{(\alpha)}=-k_{\lambda \mu}^{(\alpha)}$ and $k_{\lambda \lambda}^{(\alpha)}=0 . \quad k^{(\alpha)}=\left\{k_{\lambda \mu}^{(\alpha)}\right\}_{\lambda, \mu \in I}^{\prime} \in C^{1}(\boldsymbol{U}, \mathcal{O})$. Then $\delta k^{(\alpha)} \in Z^{2}(\boldsymbol{U}, \boldsymbol{Z})$ and for fixed $\lambda, \mu \in I\left\{k_{\lambda \mu}^{(\alpha)}\right\}_{\alpha \in A}$ is a normal family in $\Gamma\left(U_{\lambda \mu}, \mathcal{O}\right)$. By Lemmas 2 and 7 there exists $u^{(\alpha)} \in C^{1}(U, \boldsymbol{Z}) \alpha \in A$, such that $\delta u^{(\alpha)}=\delta k^{(\alpha)}$ and $\left\{u^{(\alpha)}\right\}_{\alpha \in A}$ is a relatively compact subset of $C^{1}(\boldsymbol{U}, \boldsymbol{Z})$. Let $v^{(\alpha)}=k^{(\alpha)}-u^{(\alpha)}$. Then $\left\{\boldsymbol{v}^{(\alpha)}\right\}_{\alpha \in A}$ is a relatively compact subset of $Z^{1}(\boldsymbol{U}, \mathcal{O})$. By Th. 4 there exists $w^{(\alpha)}=\left\{w_{\lambda}^{(\alpha)}\right\}_{\lambda \in I}^{\prime}$ $\in C^{0}(\boldsymbol{V}, \mathcal{O}), \alpha \in A$, such that $\delta w^{(\alpha)}=v^{(\alpha)}$ and $\left\{w^{(\alpha)}\right\}_{\alpha \in A}$ is a relatively compact subset of $C^{0}(\boldsymbol{V}, \mathcal{O})$. Define $g^{(\alpha)} \in \Gamma\left(M, \mathscr{M}^{*}\right)$ by setting $g^{(\alpha)}=f_{\lambda}^{(\alpha)} \exp \left(-2 \pi i w_{\lambda}^{(\alpha)}\right)$ on $V_{\lambda}$. Then $\left\{g^{(\alpha)}\right\}_{\alpha \in A}$ is a normal family in $\Gamma\left(M, \mathscr{M}^{*}\right)$ and $g^{(\alpha)}$ is a solution of $f^{(\alpha)}, \alpha \in A$. q. e. d.

Proof of Theorem 3. Suppose $F=\left\{f^{(\alpha)}\right\}_{\alpha \in A}$ is a small normal family of non-negative divisors on $M$. We can assume w. l.o.g. that $F$ is compact in the topological space of all non-negative divisors. There exist an open covering $\boldsymbol{U}=\left\{U_{\lambda}\right\}_{\lambda \in I}$ of $M$ and a subset $K$ of $M$ such that (1) to (5) of Def. 4 are satisfied. We can assume w. l.o.g. that $\boldsymbol{U}$ is countable. Fix $x_{\lambda} \in K \cap U_{\lambda}$ for $U_{\lambda} \neq \emptyset$. Fix $x_{\lambda / \mu}=x_{\mu \lambda} \in K \cap U_{\lambda \mu}$ for $U_{\lambda} \cap U_{\mu} \neq \emptyset$. By Ths. 1.9 (p. 168) and 2.25 (p. 188) of [8] there exists $h_{\lambda}^{(\alpha)} \in \mathrm{I}\left(U_{\lambda},(), \alpha \in A, \lambda \in I\right.$, such that (1) $h_{\lambda}^{(\alpha)}$ is a solution of $f^{(\alpha)} \mid U_{\lambda}$, (2) $h_{\lambda}^{(\alpha)}\left(x_{\lambda}\right)=1$, and (3) for fixed $\lambda \in I$ the map from $F$ to $\mathrm{I}\left(U_{\lambda},()^{\prime}\right)$ defined by $f^{(\alpha)} \rightarrow h_{\lambda}^{(\alpha)}$ is continuous.

For $U_{\lambda} \cap U_{\mu} \neq \emptyset$ choose a continuous map $\xi_{\lambda \mu}:[0,1] \rightarrow K \cap U_{\lambda}$ such that $\xi_{\lambda \mu}(0)=x_{\lambda}$ and $\xi_{\lambda \mu}(1)=x_{\lambda \mu}$. For $\alpha \in A$ and $U_{\lambda} \cap U_{\mu} \neq \emptyset$ there is a unique continuous map $\eta_{\lambda \mu}^{(\alpha)}:[0,1] \rightarrow \boldsymbol{R}$ such that $\eta_{\lambda \mu}^{(\alpha)}(0)=0$ and $\exp \left(2 \pi i \eta_{\lambda \mu}^{(\alpha)}(\theta)\right)$ $=h_{\lambda}^{(\alpha)}\left(\xi_{\lambda \mu}(\theta)\right)\left|h_{\lambda}^{(\alpha)}\left(\xi_{\lambda \mu}(\theta)\right)\right|^{-1}$ for $\theta \in[0,1]$. Let $k_{\lambda \mu}^{(\alpha)}=h_{\mu}^{(\alpha)}\left(h_{\lambda}^{(\alpha)}\right)^{-1} \mid U_{\lambda \mu}, \alpha \in A, \lambda, \mu \in I$. There is a unique holomorphic function $u_{\lambda \mu}^{(\alpha)}$ on $U_{\lambda \mu}$ such that $\exp \left(2 \pi i u_{\lambda \mu}^{(\alpha)}\right)=k_{\lambda \mu}^{(\alpha)}$ and the real part of $u_{\lambda \mu}^{(\alpha)}\left(x_{\lambda \mu}\right)$ is $\eta_{\mu \lambda}^{(\alpha)}(1)-\eta_{\lambda \mu}^{(\alpha)}(1)$. Let $u^{(\alpha)}=\left\{u_{\lambda \mu}^{(\alpha)}\right\}_{\lambda, \mu \in I}^{\prime} . \delta u^{(\alpha)} \in Z^{2}(\boldsymbol{U}, \boldsymbol{Z})$. The map from $F$ to $C^{\prime}(\boldsymbol{U}, \mathcal{O})$ defined by $f^{(\alpha)} \rightarrow u^{(\alpha)}$ is continuous. $B=\left\{\delta u^{(\alpha)}\right\}_{\alpha \in A}$ is a compact subset of $Z^{2}(\boldsymbol{U}, \boldsymbol{Z})$. By Lemma 7 there exists $a^{(\alpha)}=\left\{a_{\lambda \mu}^{(\alpha)}\right\}_{\lambda, \mu \in I}^{\prime}$ $\in C^{1}(\boldsymbol{U}, \boldsymbol{R}), \alpha \in A$, such that $\delta a^{(\alpha)}=\delta u^{(\alpha)}$ and the map from $B$ to $C^{1}(\boldsymbol{U}, \boldsymbol{R})$ defined by $\delta u^{(\alpha)} \rightarrow a^{(\alpha)}$ is continuous. Let $v^{(\alpha)}=u^{(\alpha)}-a^{(\alpha)}$. Then $C=\left\{v^{(\alpha)}\right\}_{\alpha \in A}$ is a compact subset of $Z^{1}(\boldsymbol{U}, \mathcal{O})$.

Let $c_{\lambda \mu}^{(\alpha)}=\exp \left(-2 \pi i a_{\lambda \mu}^{(\alpha)}\right)$. Then

$$
c_{\lambda \mu}^{(\alpha)} c_{\mu \nu}^{(\alpha)} c_{\nu \lambda}^{(\alpha)}=1 \text { for } U_{\lambda \mu \nu} \neq \emptyset
$$

Fix $U_{\lambda_{0}} \neq \emptyset$. Since $M$ is connected, for every $U_{\lambda} \neq \emptyset$ we can find $\lambda_{1}, \cdots, \lambda_{m} \in I$ such that $\lambda_{m}=\lambda$ and $U_{\lambda_{j}} \cap U_{\lambda_{j+1}} \neq \emptyset$ for $0 \leqq j<m$. Let $d_{\lambda}^{(\alpha)}=\prod_{j=0}^{m-1} c_{\lambda_{j j_{j+1}}}^{(\alpha)}$. Since $\pi_{1}(M)=0,(4)$ implies that $d_{\lambda}^{(\alpha)}$ is independent of the choice of $\lambda_{1}, \cdots, \lambda_{m-1}$. $d_{\mu}^{(\alpha)}\left(d_{\lambda}^{(\alpha)}\right)^{-1}=c_{\lambda \mu}^{(\alpha)}$ for $U_{\lambda \mu} \neq \emptyset$.

We can choose a locally finite open covering $\boldsymbol{V}=\left\{V_{\lambda}\right\}_{\lambda \in I}$ of $M$ such that 
$V_{\lambda}^{-}$is a compact subset of $U_{\lambda}, \lambda \in I$. By Th.4 there exists $w_{\lambda}^{(\alpha)}=\left\{w_{\lambda}^{(\alpha)}\right\}_{\lambda \in I}^{\prime}$ $\in C^{0}(\boldsymbol{V}, \mathcal{O})$ such that $\delta w^{\prime \alpha)}=v^{(\alpha)}$ on $\boldsymbol{V}$ and the map from $C$ to $C^{0}(\boldsymbol{V}, \mathcal{O})$ defined by $v^{(\alpha)} \rightarrow w^{(\alpha)}$ is continuous.

Define $g^{(\alpha)} \in \Gamma(M, \mathcal{O})$ by setting $g^{(\alpha)}=d_{\lambda}^{(\alpha)} h_{\lambda}^{(\alpha)} \exp \left(-2 \pi i w_{\lambda}^{(\alpha)}\right)$ on $V_{\lambda} \cdot g^{(\alpha)}$ is well-defined, because $\exp \left(-2 \pi i w_{\lambda}^{(\alpha)}\right) \exp \left(2 \pi i w_{\mu}^{(\alpha)}\right)=\exp \left(2 \pi i v_{\lambda \mu}^{(\alpha)}\right)=\exp \left(2 \pi i u_{\lambda \mu}^{(\alpha)}\right)$ $\exp \left(-2 \pi i a_{\lambda \mu}^{(\alpha)}\right)=k_{\lambda \mu}^{(\alpha)} c_{\lambda \mu}^{(\alpha)}=h_{\mu}^{(\alpha)}\left(h_{\lambda}^{(\alpha)}\right)^{-1} d_{\mu}^{(\alpha)}\left(d_{\lambda}^{(\alpha)}\right)^{-1}$ on $V_{\lambda \mu}$. The map $\gamma: F \rightarrow \Gamma(M, \mathcal{O})$ defined by $\gamma\left(f^{(\alpha)}\right)=g^{(\alpha)}$ satisfies the requirement. q. e.d.

Corollary. (1) holds for $M=$ the product of $\boldsymbol{C}^{q}$ and the unit ball of $\boldsymbol{C}^{p}$.

PRoOF. We can assume w. l.o.g. that $x_{0}=0$, because any point of $M$ can be mapped to the origin by some biholomorphic map of $M$ onto $M$. Let $I=\boldsymbol{N}$. For $\lambda \in I$ let $U_{\lambda}=\left\{(z, w) \in \boldsymbol{C}^{q} \times\left.\boldsymbol{C}^{p}\left|\lambda^{-2}\right| z\right|^{2}+(\lambda+1)^{2} \lambda^{-2}|w|^{2}<1\right\}$. $U_{\lambda}$ is biholomorphic to a ball in $\boldsymbol{C}^{q+p}$ and is relatively compact in $M . \boldsymbol{U}=\left\{U_{\lambda}\right\}_{\lambda \in \boldsymbol{I}}$ covers $M$. Let $K=\{0\}$. Then (1) to (5) of Def. 4 are satisfied with $F=N$. $N$ is a small normal family of non-negative divisors on $M$. q. e. d.

\section{REFERENCES}

[1] A. Aeprli, E. Calabi, AND H. RöHrL, Proceedings of the Conference on Complex Analysis (Minneapolis 1964), Springer-Verlag, New York, 1965.

[2] R.C. GunNING, AND H. Rossi, Analytic Functions of Several Complex Variables, Prentice-Hall, Englewood Cliffs, New Jersey, 1965.

[3] L. HÖRANDER, An Introduction to Complex Analysis in Several Complex Variables, D. Van Nostrand, Princeton, New Jersey, 1966.

[4] L. HÖRMANDER, Generators for some rings of analytic functions, Bull. Amer. Math. Soc., 73(1967), 943-949.

[5] G. KöTHE, Topologische Lineare Räume I, Springer-Verlag, Berlin-GöttingenHeidelberg, 1960.

[6] G. L. MCGrath, Normal families of non-negative divisors on polycylinders, Ph. D. Thesis, University of Notre Dame, Indiana, 1965.

[ 7 ] R. NARASImHan, On the homology groups of Stein spaces, Invent. Math., 2(1967), 377-385.

[8] W. STOLL, Normal families of non-negative divisors, Math. Z., 84(1964), 154-218.

MATHEMATICS DEPARTMENT

UNIVERSITY OF NOTRE DAME

Notre Dame, InDIANA, U.S. A. 